Investigaciones Fenomenológicas, vol. Monográfico 4/I (2013): Razón y vida, 247-264. e-ISSN: $1885-1088$

\title{
EXISTENCIA HUMANA, MUNDO Y RESPONSABILIDAD \\ en LA fenOMENOLOgÍA de JAN PATOČKA *
}

\author{
HumAN EXISTENCE, WORLD AND RESPONSIBILITY \\ in the Phenomenology of Jan Patočka
}

\author{
Iván Ortega Rodríguez \\ Fenomenología y Filosofía Primera/ \\ Universidad Pontificia de Comillas, España \\ ivan.ortega79@gmail.com
}

\begin{abstract}
Resumen: En este trabajo buscamos dar cuenta de la evolución y continuidad de la fenomenología de Jan Patočka en torno al tema del mundo y relación de la existencia humana con el mismo. Creemos que este problema subyace y anima toda su investigación fenomenológica y que es clave para comprender el conjunto de su pensamiento.
\end{abstract}

Palabras clave: Jan Patočka, fenomenología, ontología, filosofía de la existencia humana.

\begin{abstract}
In this paper we seek to take notice of the evolution and continuity of Jan Patočka's phenomenology on the topic of the world and human existence's relationship with it. We believe that this problem underlies and stimulates Patočka's whole phenomenological research and we think that it is a key element to understand the ensemble of his thought.
\end{abstract}

Key Words: Jan Patočka, Phenomenology, Ontology, Philosophy of Human Existence.

\section{MUNDO Y EXISTENCIA HUMANA COMO PROBLEMA MOTOR EN LA EVOLUCIÓN DE PATOČKA}

Uno de los rasgos más inmediatamente destacables de la obra fenomenológica de Jan Patočka es la notable diferencia entre los textos escritos en su juventud y los que elaboró más tarde. Los primeros se sitúan decididamente dentro de la fenomenología subjetiva trascendental de Edmund

\footnotetext{
* Este texto está basado en una ponencia leída en la IV Conferencia Mundial de Fenomenología: Razón y Vida. La Responsabilidad de la Filosofía, Segovia, 19-23 de septiembre de 2011, con el titulo "A Line of Continuity in Jan Patočka's Phenomenological Thought". Ha habido, empero, modificaciones sustanciales, de manera que el texto que aquí presentamos es notablemente distinto; no toma en cuenta algunos puntos allí abordados y, en cambio, profundiza en algunos que sí se presentaron y aborda otros que en ella no estuvieron presentes. La ponencia se encuentra publicada, con traducción propia y modificaciones de menor rango, en Pensamiento, cf. "Una línea de continuidad en la fenomenología de Jan Patočka", en Pensamiento, 69 (2013), 301-313.
} 
Husserl. Sin embargo, posteriormente el panorama es muy distinto, pues se nos habla de existencia finita, de intersubjetividad en un mundo compartido, de movimiento de la existencia y del proyecto de una "fenomenología asubjetiva". A primera vista parece que poco tienen que ver. Una radical cesura parece separar ambos periodos. Esta separación, además, puede explicarse por las influencias recibidas y puede, incluso, localizarse cronológicamente con cierta precisión. En efecto, si en sus primeros años Patočka da por bueno el marco husserliano de una fenomenología subjetiva, con relevantes influencias de otros autores como Bergson, posteriormente el autor clave parece ser Heidegger y su concepción de la existencia como finita y lugar de mostración del ser. Dicho cambio, además, se produce hacia mediados de los años 40 del siglo pasado, con seguridad antes de 1947, fecha de publicación de Eternidad e historicidad, donde hay ya una crítica clara de la fenomenología trascendental ${ }^{1}$. Parecería, pues, que hay dos proyectos fenomenológicos radicalmente diferentes.

No cabe duda de que hay mucho de verdad en esta descripción. Sin embargo, es igualmente cierto que hay una continuidad en la obra de Patočka, hecha de temas y preocupaciones recurrentes que conforman un problema fundamental que anima su investigación. Es cierto, empero, que esta continuidad no es inmediatamente visible, pues su obra ofrece una enorme variedad de perspectivas en diversos campos. En efecto, el lector de Patočka encuentra notables dificultades a la hora de dar una interpretación unitaria de sus planteamientos. Esta dificultad se presenta, primeramente, cuando tiene que dar cuenta de la totalidad de su trabajo, pero también cuando tiene que indicar cuál es el núcleo de alguna de sus diversas áreas de investigación fenomenología, filosofía de la historia, estética, Comenio, la historia de los checos...-. No es de extrañar, pues, que haya quien niegue unidad alguna en su obra, es el caso del filósofo checo Petr Rezek².

La opinión mayoritaria, sin embargo, sí cree que hay una unidad fundamental en toda su obra; eso sí, se trata de una unidad centrada en temas y preocupaciones, no en la de un armazón conceptual sistemático. En cuanto a

${ }^{1}$ Cf. J. Patočka Večnost a dejjinnost, Oykoimenh, Praga, 2007. Esta última edición incluye el plan de la obra y adjunta un ensayo titulado El humanismo checo y su última palabra en Rádl ("Ceský humanismus a jeho poslední slovo v Rádlovi", en Večnost a dějinnost, pp.11-17). Hay traducción francesa, sobre la base de la edición separada de 2007: Eternité et historicité, traducción de Erika Abrams, París, Verdier, 2011.

${ }^{2}$ Cf. Josef Moural, "The Question of the Core of Patocka's Work: Phenomenology, History of Philosophy, and Philosophy of History", Praga, documentos del CTS, CTS-99-05, marzo de 1999. 
la fenomenología, se ha dicho que el núcleo de su indagación es el esclarecimiento fenomenológico del mundo, esto es, cómo rendir cuenta del mundo en su donación y las consecuencias de concebir la misma de uno u otro modo $^{3}$. Nosotros reconocemos la validez de esta descripción. No obstante, nos permitimos una pequeña modificación que precisa el sentido de este problema central. Así, nosotros creemos que el elemento clave es el de la relación entre subjetividad y mundo -o entre existencia humana y mundo, que en nuestro autor son intercambiables ${ }^{4}-$. Patočka, ciertamente, pivota en torno al problema de cómo dar cuenta del mundo. Pero al dar cuenta del mismo, toma constantemente en consideración el darse del mundo y su análisis fenomenológico -esto es, el análisis de su hacerse fenómeno, de su mostrarse-; y por ello, toma en cuenta también la subjetividad y la relación de la misma con el mundo ${ }^{5}$. Por otro lado, la subjetividad y su relación con el mundo son también una preocupación constante en tanto Patočka busca dar cuenta de cómo la subjetividad se comporta respecto del mundo y cómo vive y actúa en relación con el mismo, así como las posibilidades y limitaciones de dicha acción. En especial, al trabajar esta cuestión en sus diversos escritos, Patočka considera las posibilidades de una vida autorresponsable, ideal que él considera nuclear del modo de vida filosófico, haciendo suya la posición husserliana ${ }^{6}$. Esto válido primeramente en su fenomenología pero también para

\footnotetext{
${ }^{3}$ Cf.M. Bernard, "Le monde comme problème philosophique", en Les études philosophiques, n³, 2011, pp. 351-373.

${ }_{4}^{4}$ En efecto, desde muy pronto Patocka describe la subjetividad como existencia, , incluso cuando defiende una fenomenología trascendental subjetiva. Sólo en su tesis doctoral de 1931, cuando todavía sus líneas básicas no habían terminado de forjarse, puede decirse que no entiende la subjetividad como existencia ( $y$ existencia con rasgos concretos, por más que ello pudiera chocar con su intento de describir una subjetividad trascendental, asunto que algunos creen que contribuye a que Patocka abandone posteriormente la fenomenología en línea husserliana).

${ }^{5}$ Otros autores coinciden con nuestra interpretación. Cf., por ejemplo, Karel Novotný, "Corps, corps propre et affectivité de I'homme", en Les études philosophiques, n03, 2011, pp.351-373. Algunos incluyen explícitamente la relación con los otros y el hecho de que se vive en un mundo compartido; y ello, a su vez, se une al tema del "cuidado del alma" (cf., por ejemplo, E.F.Findlay, Caring for the Soul in a Postmodern Age. Politics and Phenomenology in the Thought of Jan Patocka, New York, State University of New York Press, 2002, pp. 15-50. coincidimos con ellos en lo nuclear de estas cuestiones, si bien, a efectos de formulación, preferimos mantener como tema el de la relación de la subjetividad y mundo, mientras que los temas de la intersubjetividad y mundo compartido los tomamos como despliegues inmediatos al desarrollar el problema. El tema del cuidado del alma, a su vez, igualmente puede entenderse como asunto nuclear de nuestro filósofo, pero nosotros lo interpretamos como un tema que resume el problema de una existencia humana autorresponsable en el mundo, problema que se plantea a partir de la pregunta por cómo la subjetividad se sitúa respecto del mundo.

${ }^{6}$ Esta cuestión central, asimismo, ha de entenderse en un sentido muy concreto. No ha de entenderse que éste es el asunto que Patočka tenga por tema central o explícito en todas sus investigaciones. Éstos giran en torno a motivos diversos, como la confrontación crítica con Husserl o Heidegger, el espacio, el mundo natural, la existencia como movimiento, el cuerpo, etc. Lo que queremos decir al afirmar como tema central la existencia en el mundo, es que esta cuestión es el problema filosófico que subyace a todas sus cuestiones y que las motiva. Si se permite la expresión, la relación entre existencia humana y
} 
el conjunto de su obra, por cuanto este problema básico motiva sus reflexiones en los diferentes campos.

En este trabajo queremos ocuparnos de este problema central, de la presencia constante del mismo, de los cambios en su descripción y de los rasgos que permanecen, así como las implicaciones para el problema de la libertad y la responsabilidad. Nuestra exposición se mueve dentro de su investigación fenomenológica, aunque queremos indicar también su relevancia para cuestiones centrales de su filosofía de la historia, que a su vez tiene no poca importancia para pensar hoy la posibilidad de una filosofía de la libertad y la responsabilidad desde un punto de partida fenomenológico.

\section{EXISTENCIA hUMANA Y MUNDO EN LA FENOMENOLOGÍA TRASCENDENTAL "SUBJETIVA"}

El problema de la relación entre existencia humana y mundo subyace, pues, al conjunto de su trabajo. Una primera fase aborda el problema, como hemos dicho, en las coordenadas de la fenomenología trascendental subjetiva de Husserl. Dentro de esta primera fase, a su vez, hay dos momentos diferenciables. El primero comprende los años 1931-1936; las obras principales aquí son su tesis doctoral y su tesis de habilitación. La primera, El concepto de evidencia y su relevancia para la noética ${ }^{7}$ es un trabajo de un Patočka aún muy joven, de unos 24 años. En él, nos encontramos con un filósofo aún en formación, que recoge las principales influencias que ha tenido hasta el momento, aventura sus primeras respuestas y apunta ya sus temas fundamentales. Y ahí, en esta forma aún inmadura en el mejor sentido de la palabra, advertimos ya el tema del mundo y la relación de la subjetividad con el mismo. Así, Patočka, en su "esbozo de las "estructuras evidentes de nuestro mundo" menciona en primer lugar la "distinción fundamental y la correlación sujeto-objeto" ${ }^{\prime 8}$. Además, el problema de la relación entre subjetividad y mundo se muestra central en un sentido más profundo, en un plano que sostiene el trabajo entero, a saber, en el mismo concepto de evidencia y la centralidad que

mundo es el tema en torno al que "damos vueltas" mientras se abordan las diferentes cuestiones y desde el que surgen los diversos desarrollos de Patočka.

7 Cf. J. Patočka "Pojem evidence a jeho vyznam pro noetiku", in Sebrané Spisy 6, Fenomenologické Spisy 1, Praha, Oikoymenh, pp.15-125.

8 Ibid, p.34. 
le da Patočka para la noética y la filosofía en general. En efecto, si la filosofía debe basarse en principios cuya verdad sea cierta y dicha certeza se ha de atestar en la evidencia, entonces no podemos sino emplazarnos en la subjetividad que conoce y aquello que conoce, que, ya entonces, viene dado para Patočka como mundo.

En su tesis de habilitación, El mundo natural como problema filosófico ${ }^{9}$, de 1936, Patočka plantea que la solución al problema de la división entre el mundo de la vida y el mundo descrito por las ciencias naturales descansa en la común referencia a la subjetividad trascendental. De esta manera, nos encontramos en su exposición con el mundo y la subjetividad como polos básicos en torno a los cuales ha de pensarse un problema determinado, como, en este caso, el de la unidad perdida del mundo humano. Su tesis de habilitación, asimismo, muestra un modo de trabajar que es igualmente constante en Patočka: la consideración de un problema urgente, que afecta a nuestra vida, la afirmación de un enfoque filosófico del mismo, y la remisión a un problema filosófico de fondo. Asimismo, también en su tesis de habilitación encontramos otra constante de su pensar, ahora referida a los contenidos, según la cual toma un problema urgente como el de la crisis contemporánea - en lo relativo a la tecnificación-, afirma la necesidad de la filosofía para determinar sus raíces y solución, y remite al problema del mundo y la relación de la subjetividad con él.

También se mueve dentro del horizonte de la fenomenología trascendental una investigación que Patočka desarrolla en los años de la Segunda Guerra Mundial, que los editores de las obras de Patočka han titulado Lo interior y el mundo ${ }^{10}$. En estos textos, se afirma de nuevo el aparecer de mundo dentro de una subjetividad como dato fundamental, con la variante de que la subjetividad viene caracterizada, en una medida mayor, por una "interioridad" que se presenta como nota de la misma. Asimismo, estos textos preludian otro aspecto de la obra de Patočka, como es el de la relación de la investigación sobre el mundo y la subjetividad con el esclarecimiento filosófico de la historia europea, en especial, con la transformación dada a partir de la modernidad. En efecto, los manuscritos de Lo interior $y$ el mundo forman parte de un proyecto

9 Cf. J.Patočka, "Přirozený svět jako filosofický problém", en Sebrané Spisy 6, pp.129-261. [Le monde naturel comme problème philosophique, traducción de Jaromír Daněk y Henri Déclève, La Haya, Martinus Nijhoff, 1976]

10 "Das Innere und die Welt", traducción de Sandra Lehmann, con una introducción de Ana Santos, en: Studia Phaenomenologica VII (2007) 15-70. 
mayor en el que nuestro autor buscaba el tránsito del "hombre cristiano" de la Edad Media al "hombre poscristiano" de la Modernidad en adelante. Este trabajo quedó incompleto, por lo que no resulta posible determinar su resultado final; sin embargo, sí resulta claro que la investigación sobre la interioridad de la vida subjetiva y la constitución del mundo en ella iban a ser la base filosófica que le daría inteligibilidad profunda a sus investigaciones propiamente históricas. Y una vez más, las posiciones y análisis concretos de Patočka variarán a lo largo de su vida, pero el mundo y su relación con la subjetividad siguen siendo el problema básico que subyace a las consideraciones de filosofía de la historia; e igualmente, el método para abordar este problema es, y será, el fenomenológico ${ }^{11}$.

\section{EL MOVIMIENTO DE LA EXISTENCIA HUMANA Y EL MOVIMIENTO DEL MUNDO}

Cuando Patočka retoma explícitamente el trabajo en fenomenología, lo hace de una manera netamente diferente a sus primeros años. Como ya se ha indicado, el mundo, y lo que en él aparece, no viene referido últimamente a la subjetividad trascendental y la tarea fenomenológica no es la de reconducir el fenómeno a la esfera subjetiva. En su lugar, Patočka explora las posibilidades de una refundación "asubjetiva" de la fenomenología ${ }^{12}$. Elegimos conscientemente el verbo "explorar" porque la investigación de Patočka es eminentemente tentativa; inquiere diversas maneras de realizar una fenomenología alejada del subjetivismo, sin que se llegue a algún resultado que se pueda tener por "el" sistema de Patočka. No obstante, y sobre la base del problema fundamental de la subjetividad y el mundo, sí que es posible determinar algunos rasgos elementales que se repiten en sus diversas tentativas. Estos rasgos, con todo, tampoco indican un solo proceder sino que

\footnotetext{
${ }^{11}$ Cf. F.Karfík, "Patockova strahovská pozưstalost a jeho odložené opus grande", en Kritický sborník, XX (2000/2001) 125-160. ["Jan Patockas Strahov-Nachlass und sein unvollendetes opus grande", en L.Hagedorn (ed.), Jan Patocka Andere Wege in die Moderne. Studien zur Europaïschen Ideengeschichte von der Renaissance bis zur Romantik, Würzburg, Königshausen \& Neumann, 2006, pp.31-63]

12 Por fortuna, una buena parte de los textos relevantes está disponible en castellano, en la reciente compilación de A.Serrano de Haro (El movimiento de la existencia humana, Encuentro, Madrid, 2004, 283 pp.). Otros se encuentran en una recopilación en francés titulada Qu'est-ce que la phénoménologie? (traducción de Erika Abrams, Jerôme Millon, Grenoble, 2002). En castellano también puede consultarse con provecho la traducción de unas lecciones de Patočka en la Universidad Carolina de Praga (en el breve periodo en que pudo enseñar públicamente), Introducción a la fenomenología, traducción de Juan A.Sánchez, revisión de Iván Ortega, Herder, Barcelona, 2005. Además de introducir la obra de Husserl y la de Heidegger, anade Patočka comentarios que van en la línea de la fenomenología asubjetiva que aquí tratamos.
} 
- si nuestra lectura es correcta - apuntan a dos caminos que Patočka explora para dar cuenta del fenómeno sin remitir a una subjetividad trascendental. Estos dos caminos no son incompatibles, y puede incluso sostenerse que convergen desde el punto de vista lógico y a partir de los textos de Patočka, pero ello puede decirse al tiempo que se debe afirmar la diferencia entre ambas vías.

Así pues, el primero de estos caminos, que Patočka emprende al menos desde 1960, parte de la consideración de la existencia como movimiento, que es parte del movimiento general del mundo, un movimiento, a su vez, que ha de entenderse en un sentido que Patočka elabora a partir de una original lectura de Aristóteles $^{13}$. La existencia humana, en efecto, debe entenderse como movimiento en el sentido aristotélico de autorrealización ${ }^{14}$. Sin embargo, dado que, siguiendo a Heidegger, Patočka afirma que esta existencia es igualmente la apertura al ser en el realizar posibilidades, esto es, que su esencia consiste en la existencia así entendida, no puede aceptarse la tesis de un sustrato permanente del movimiento. Ello es así, fundamentalmente, porque asumir dicho sustrato permanente supondría afirmar al existente humano como un ente e implicaría, por tanto, situarnos de nuevo en el nivel del ente, considerando así que la apertura al ser que es la existencia es la de un ente respecto de otro ente. Sin embargo, Patočka considera que si prescindimos del sustrato permanente, entonces la noción aristotélica de movimiento como autorrealización sí se ajusta para describir la trama misma de la existencia humana como realización de posibilidades y apertura del ser. Así, la existencia humana es un movimiento vital de autorrealización por el que diferentes atributos se van reuniendo al existente humano que, de este modo, se va

\footnotetext{
${ }^{13}$ Entre las obras disponibles en castellano que reflejan este camino podemos citar "El mundo natural y la fenomenología", en El movimiento de la existencia humana, pp.13-56 y "Universo y mundo del hombre. Observaciones a un planteamiento cosmológico contemporáneo", en ibídem, pp.85-92 (en este último, por cierto, encontramos apoyo textual para la convergencia de los dos caminos, cf.pp.90s.). Con todo, la exposición más completa de este camino está en sus lecciones sobre la corporalidad. Cf. J. Patočka, Body, Community, Language, World, traducción de Erazim Kohák, Chicago, Open Court, 1998 y "Leçons sur la corporéité", en Papiers Phénoménologiques, Grenoble, Jerôme Millon, 1995, pp.53-116. El texto en inglés procede de las notas de sus alumnos y el francés son las notas para clase del propio Patočka. Como estudio, podemos citar el excelente trabajo de F.Jacquet, "Vie et existence. Vers une cosmologie phénoménologique", en Les études philosophiques, 3/2011, pp.395-419.

${ }^{14}$ Sobre la lectura de Aristóteles por parte de Patočka, Cf. M.Larison, "Du mouvement chez Aristote d'après Jan Patočka", en N.Frogneux (dir.), Jan Patočka. Liberté, existence et monde commun, Bruselas, Le Cercle Herméneutique, 2012, pp.179-93. La obra más importante donde Patočka interpreta a Aristóteles es Aristóteles, sus predecesores y sucesores, cf.J. Patočka, Aristote, ses devanciers, ses succeseurs, traducción de Erika Abrams, París, Vrin, 2011. Especialmente el capítulo 3 (pp.101-262), una selección del mismo se ha publicado aparte, cf. "La science philosophique de la nature chez Aristote (extrait)", en Les études philosophiques 3/2011, pp.303-30.
} 
conformando ${ }^{15}$. De todos los cambios descritos por Aristóteles, el caso principal para Patočka es el de la generación y corrupción, pues ahí la transformación no se da entre un ente con unas determinadas cualidades a ese mismo ente con otras, sino que se pasa de no haber ente en absoluto a haberlo, y viceversa. En este tipo de transformación, lo que tenemos es verdaderamente la autoconstitución o autorrealización del ente. Así, Patočka piensa que este movimiento de transformación y advenimiento ${ }^{16}$ de propiedades, en el sentido más radical y propio de autorrealización, es el que mejor explica la trama íntima de la existencia humana como realización de sí asumiendo posibilidades. La esencia del Dasein heideggeriano, que es la existencia, es, en su articulación interna, movimiento aristotélico de autorrealización, con la salvedad referida al sustrato permanente ${ }^{17}$.

El movimiento de la existencia humana, asimismo, es uno, pero no uniforme, pues se diversifica en tres modalidades, desplegadas en la llamada "teoría de los tres movimientos de la existencia humana", de amplias repercusiones en filosofía de la historia y que, con justicia, es uno de los aspectos más conocidos del pensamiento de Patočka. Por otro lado, el movimiento de la existencia es correlativo de otro movimiento, igualmente de autorrealización, que es el movimiento de constitución del mundo, por el cual el mundo y los diferentes entes vienen a ser $-\mathrm{y}$ a aparecer, primero de forma no manifiesta y luego a la conciencia-. Es más, la existencia humana se muestra, en último análisis, como siendo parte de este movimiento general, que algunos han descrito acertadamente como movimiento ontogenético (o incluso, si se nos permite el término, cosmo-onto-genético). En definitiva, Patočka asume la noción aristotélica de movimiento para repensar en profundidad la subjetividad y el aparecer de mundo a la misma, y esto le hace llegar a una compleja cosmología fenomenológica que resulta ciertamente llamativa y que contrasta

\footnotetext{
${ }^{15}$ Queda pendiente la cuestión de si afirmamos un sustrato no permanente, que se va constituyendo y cambiando con el movimiento existencial, o si renunciamos a todo sustrato y afirmamos un cierto correlacionismo dinámico de atributos que se vinculan. Patočka parece oscilar entre ambas posibilidades, aunque parece tener más fuerza la de un sustrato móvil, por cuanto estima que el movimiento de autorrealización presupone un espacio y una magnitud, esto es, una corporalidad. Cf.M.Larison, art.cit., pp.191-3.

${ }^{16}$ El término "advenimiento" no es empleado propiamente por Patočka, pero sí utiliza expresiones que hablan de que las posibilidades "vienen a mí" desde el mundo, cf., por ejemplo, Papiers Phénoménologiques, op.cit., pp. 123-4. Por esta razón algunos intérpretes de lengua francesa han querido acercar incluso terminológicamente la fenomenología de Patočka a la de Claude Romano, empleando a propósito expresiones como "avènement", "événement", "phénoménologie événementielle", etc. Cf. E.Tardivel, La liberté au príncipe. Essai sur la philosophie de Patočka, París, Vrin, 2011, pp.149-163

${ }_{17}$ Cf. R.Barbaras, Vie et intentionnalité. Recherches phénoménologiques, Paris, Vrin, 2003, pp.6-23.
} 
con sus primeros trabajos.

Por lo demás, una apretada exposición como ésta no puede entrar en todos los detalles de la teorización de Patočka sobre el movimiento de la existencia y el movimiento del mundo. Señalemos, con todo, algunos aspectos que complementan lo aquí presentado. Así, hemos dicho que, en el movimiento del mundo, los entes vienen a ser "y aparecer" en dos ocasiones, una "implícita" y la otra "a la conciencia". Evidentemente, dentro de una investigación fenomenológica, tiene que llamar la atención que se hable de tal manifestación "implícita". Ella viene afirmada como necesariamente previa a la manifestación a la conciencia y consiste en la primera delimitación de un ente a partir del movimiento general cosmológico. Obviamente, este primer aparecer sólo puede ser incluido en una descripción fenomenológica si puede ser atestado, de algún modo, en el análisis fenomenológico, cosa que Patočka apunta en algunos de sus escritos cuando parte del movimiento de la existencia para afirmar el movimiento del mundo ${ }^{18}$. Por otra parte, hay un problema con la continuidad entre el movimiento de la existencia y el movimiento cosmológico, pues por una parte se afirma que, en última instancia, son el mismo movimiento, pero por otra la caracterización del movimiento de la existencia humana como denotado por la manifestación y la distancia respecto de lo dado en la misma especialmente en el tercer movimiento- invitan a verlo más bien como rigurosamente diferente del movimiento cosmológico. Esta tensión, por lo demás, parece estar presente en las descripciones del mismo Patočka y guardan, asimismo, clara relación con otra tensión dentro de la teoría de los tres movimientos, a saber, la que se da entre el primer y segundo movimiento, por un lado, y el tercero, por otro, que lleva unas veces a oponerlos dicotómicamente y otras a ver una cierta continuidad. Estas dos tensiones no son necesariamente idénticas, pero queda claro que en los dos casos tratamos con una dialéctica fundamental entre identificación con el mundo en tanto ámbito del que procedemos y en el que venimos dados, por una parte, y distanciamiento del mismo, por otra.

En cualquier caso, queda claro que el problema fundamental sobre el que

\footnotetext{
${ }^{18}$ Estas afirmaciones se encuentran dispersas por sus escritos, de entre los publicados podemos señalar "Problém přirozeného světa", en Tělo, společenství, jazy, svět, Praga, Oikoymenh, 1995, pp.201-2. ["El problema del mundo natural", en Cuerpo, comunidad, lenguaje mundo]. No hay, que sepamos, traducción a otras lenguas.
} 
viene motivada toda esta investigación del movimiento de la existencia y del mundo, es el del mundo y la subjetividad en su relación mutua. El complejo camino que sigue Patočka entre Aristóteles y la fenomenología en esta filosofía del existente finito y del dinamismo universal atesta, finalmente, una profunda meditación del mismo tema de fondo que le ocupa desde su juventud. Y más allá del interés relativo a la biografía intelectual del autor, estos complejos caminos intelectuales hablan elocuentemente de un problema presente "en la cosa misma" de la relación entre subjetividad y mundo que ha de estimular, ciertamente, la investigación fenomenológica.

\section{EXISTENCIA hUMANA Y MUNDO EN LA FENOMENOLOGÍA DEL "APARECER EN CUANTO TAL"}

Parecido interés tiene el segundo camino por el que explora una fenomenología liberada del marco trascendental subjetivo. Cabe caracterizarlo como la fenomenología del "aparecer en cuanto tal". Iniciado posteriormente, hacia 1970, este proyecto busca determinar una "auto-atestación" del fenómeno, donde el aparecer dé cuenta de sí sin que deba remitir su constitución a una subjetividad - aunque, como hemos dicho, el análisis deba iniciarse en el sujeto-. Si hemos de caracterizar sucintamente este camino, podemos decir que su punto de partida es tomar el fenómeno como "aparecer en cuanto tal", excluyendo que sea algún tipo de subjetividad trascendental; a partir de ahí, se afirma que el tema propio de la fenomenología es el estudio de esta esfera del aparecer. A su vez, el método para acceder a este "aparecer en cuanto tal" desde la fenomenología pasa, principalmente, por darle primacía a la epojé sobre la reducción y efectuarla de modo radical, sin excluir la subjetividad. De este modo, se afirma una esfera autónoma de aparecer con sus propias leyes, se la caracteriza como mundo y en ella la subjetividad es un polo necesario de referencia, pero en modo alguno es constituyente sino, al contrario, finita y constituida por el mundo.

Así, el fenómeno, considerado rectamente, ha de concebirse como "aparecer en cuanto tal". Para Patočka, si nos atenemos rigurosamente a lo dado, lo que encontramos es la donación misma, es el "darse" en cada caso de "algo" a "alguien", donde lo central es la apertura misma, la donación; en ella, 
la subjetividad es parte del dato, es un polo de referencia, pero no sustrato último de la misma en sentido alguno ${ }^{19}$. Patočka concibe esta apertura como aparecer, como estarse dando, un momento imprescindible para todo comportamiento teórico y práctico, generalmente pasado por alto en la ocupación vital con lo que aparece y viene abierto por este aparecer que, así, queda en el olvido ${ }^{20}$. Y analizando este punto de partida de la apertura como aparecer o estarse dando, llegamos a que este aparecer puede ser tomado "en sí mismo", como algo analizable desde sí, como un dato en el que nos podemos detener $\mathrm{y}$, en cierto modo, "quedarnos dentro" para analizarlo en sus estructuras. Patočka se esfuerza en mostrar que el fenómeno, al analizarlo en lo que es dado y sólo en tanto dado - siendo realmente fieles, nos dice, al "principio de todos los principios"-, abre, en realidad, a la esfera del aparecer como algo analizable en sí mismo, aunque refiera a la subjetividad como uno de sus polos ${ }^{21}$. En modo alguno esta esfera del aparecer puede ser verdaderamente caracterizada como una subjetividad, como una "esfera egológica". Ahora bien, ¿cómo se puede acceder, en un análisis fenomenológico a esta esfera del aparecer?

El principal camino explorado por Patočka es el de la epojé universal. La epojé tiene para Patočka un alcance mayor que el que le dio Husserl ${ }^{22}$. En efecto, Patočka critica a Husserl que subordinara la epojé a la reducción. Así,

\footnotetext{
${ }^{19}$ Los trabajos más importantes en este proyecto de fenomenología del aparecer en cuanto tal están reunidos en El movimiento de la existencia humana. Se trata de "El subjetivismo de la fenomenología husserliana y la posibilidad de una fenomenología 'asubjetiva'"; "El subjetivismo de la fenomenología husserliana y la exigencia de una fenomenología asubjetiva"; y "Epojé y reducción", pp.93-112; 113113-35; 241-50 respectivamente.

${ }^{20}$ Cf. Platon et l'Europe, traducción de Erika Abrams, París, Verdier, 1992, pp. Aunque el contexto es el de unos seminarios cuyo tema es la filosofía platónica, Patočka se detiene a explicar el "enigma" del aparecer como elemento que suscita el origen del ideal de vida en verdad y la filosofía.

${ }^{21}$ La referencia a algunos textos puede aclarar la cuestión. Por un lado, en un texto divulgativo de la fenomenología trascendental, Las conferencias de Londres, Husserl afirma con claridad que "La formulación del no ser del mundo (o el abstenerse de tomar partido en relación con las dos posibilidades de ser o no ser del mundo) conduce, cuando yo reflexiono, a la evidencia absoluta y apodíctica 'tengo estas y aquellas experiencias naturales, veo esta casa', al mismo tiempo que dejo abierto el ser de las casas [...] pero este absoluto 'yo experimento esta casa estas calles, etcétera' no es todo. Ahora tropiezo, instantáneamente, con unas corrientes de vivencias muy diversas, con el ego cogito concreto. [...] Todo esto recibe su sentido absoluto como un ser que fluye continua y absolutamente si, reflexionando, lo tomo en su ser propio y esencial, en esta epogé" (cf. Las conferencias de Londres, traducción de Ramsés Soberano, Salamanca, Sígueme, 2012, p.38). En cambio, Patočka afirma lo siguiente: "Y naturalmente estos caracteres téticos y caracteres del darse son un índice de que lo que aparece, aparece para alguien [...] Pero lo que nosotros cuestionamos es el derecho de que aquello por lo cual aparece lo que aparece se convierta en un objeto ulterior de una posible 'percepción interna' que lo capte 'en el original'. [...] Hay un campo fenoménico, un ser del fenómeno en cuanto tal, que no puede reducirse a ningún ente que aparece en él, que no puede explicarse nunca por el ente, sea de índole objetiva al modo de la naturaleza o subjetiva al modo del yo" ("El subjetivismo de la fenomenología de Edmund Husserl y la exigencia de una fenomenología asubjetiva", pp-127 y 129. Hemos modificado ligeramente la redacción. El subrayado es nuestro).

22 Cf. J. Patočka, "Epojé y reducción", en El movimiento de la existencia humana, pp.241-50.
} 
Husserl le da primacía a la subjetividad y la epojé está ordenada al requerimiento de reconducir los fenómenos a la subjetividad trascendental. Por esta razón, la epojé no puede aplicarse a la subjetividad, pues significaría paralizar el camino de reducción. Sin embargo, esta subordinación se debe, a ojos de Patočka, a un prejuicio no justificado, derivado de identificar la evidencia del sujeto en su existencia (el sum), la evidencia del hecho de que existe, con la evidencia de un sujeto que sostiene últimamente el darse de todo lo que aparece. En efecto, una cosa es reconocer que el aparecer lo es a un sujeto y que éste viene dado a sí mismo con una existencia indudable, y otra es dar por evidente que este sujeto es el fundamento del aparecer de lo dado ${ }^{23}$. En cambio, si se prescinde del presupuesto de este sujeto trascendental y se efectúa esta epojé radical, lo que tiene lugar está lejos de ser una parálisis de la investigación. Al contrario, nos encontramos ante el aparecer mismo, ante el darse de todo lo que viene dado en fenómenos, incluyendo la subjetividad ${ }^{24}$. Es más, es el aparecer el que posibilita la aparición del yo, y no al revés ${ }^{25}$. Lo que nos queda al descubierto es el aparecer mismo, en su doble dimensión de "hacer aparecer" las cosas y hacer que aparezcan "al" sujeto, el cual, a su vez, aparece ante sí mismo en este aparecérsele las cosas.

Por su parte, la esfera del aparecer presenta una estructura en tres polos: la subjetividad (en realidad, subjetividades), esto es, el "a quién" aparece; los objetos y el mundo como totalidad de objetos, "lo que" aparece; y las leyes propias del aparecer, los reenvíos de un dato a otro que se dan entre los diversos fenómenos y que no son leyes atribuibles a los objetos mismos (el "cómo" aparece) ${ }^{26}$. Asimismo, esta esfera de aparecer es caracterizada globalmente por Patočka como "mundo", que no debe confundirse con el

\footnotetext{
${ }^{23}$ Cf. J.Patočka, "El subjetivismo de la fenomenología de Edmund Husserl y la exigencia de una fenomenología asubjetiva", p.133. Por otro lado, Patočka sabía bien que Husserl se refería a algo distinto a un yo empírico, pues la "esfera egológica" viene dada una vez se ha suspendido la aplicación tesis de la existencia del mundo, pero aún así piensa que este "yo" tan peculiar del que habla Husserl sigue siendo fruto de un añadido no fenomenológico.

${ }^{24}$ Cf. J.Patočka, "Epojé y reducción", op.cit., pp.247-50. En esas páginas se dice, por ejemplo: "Entendida así, la epojé no es un acceso a ningún ente o pre-ente, sea mundano o no mundano, pero justamente por ello es quizás el acceso al aparecer en lugar de a lo que aparece, esto es, al aparecer en cuanto tal" (p.247)

25 "Creer que el aparecer sea alguna cosa que necesitaría sujetos como soporte y fundamento, es un prejuicio; quizás ocurra al revés, que los sujetos no sean posibles a menos que haya el plano de la aparición que hace posible algo así como una referencia a sí mismo, puesto que el referirse-a-sí-mismo presupone un aparecer a sí mismo". J. Patočka, "[Corps, possibilités, monde, champ d'apparition], en Papiers Phénoménologiques, op.cit., pp.117-129, p.129. El segundo subrayado es nuestro.

${ }^{26}$ Cf. J.Patočka, "[Epoché et Réduction: manuscrit de travail]", en Papiers Phénoménologiques, op.cit., 163-211, especialmente la p.177.
} 
mundo como totalidad de objetos, sino que se le ha de entender como horizonte omniabarcante de todos los horizontes de aparición ${ }^{27}$. El aparecer se da dentro de horizontes, en los que lo dado remite en cada caso a algo que no está dado inmediatamente pero que viene incluido en el dato y que abarca ambos modos de dato - el inmediato y el dado en su ausencia-; y en último término, este conjunto de horizontes, en el seno de los cuales se dan los reenvíos de la esfera del aparecer, remite a un horizonte de horizontes que los abarca a todos y que es el mundo ${ }^{28}$. Así, el mundo, en este sentido de horizonte de horizontes, se convierte en una auténtica esfera a la que todo aparecer $-y$, con él, todo lo que aparece- viene finalmente referido. Puede decirse que el mundo como horizonte ocupa el lugar que en Husserl tenía la subjetividad trascendental, por lo que se ha dicho acertadamente que para Patočka el trascendental es el mundo.

En todo ello, puede verse que el tema de fondo sigue siendo el mundo y la existencia humana. La transformación que ha tomado la teorización es aquí, al igual que en la filosofía del movimiento de la existencia, más que notable. Con todo, el tema que late de fondo, el que sigue animando la investigación, es el del mundo dándose a la subjetividad -en realidad, intersubjetividad ${ }^{29}$ Asimismo, una investigación detenida puede hacernos ver que también hay temas concretos que han permanecido al par que han sufrido una transformación. Es el caso del motivo del "horizonte" que acabamos de mencionar; en efecto, ya en sus primeros años, y plenamente dentro del marco trascendental, Patočka considera que el aparecer se da en horizontes, que vienen dados en virtud de una "intencionalidad de horizonte" que la que da el objeto, y que el mundo es el horizonte de horizontes ${ }^{30}$. La diferencia, claro

27 Así: "Ciertamente lo yoico no es nunca percibido en y por él mismo, o experimentado inmediatamente como quien quiera que sea, sino sólo como centro de organización de una estructura universal de aparición que no es reductible a lo que aparece como tal en su ser singular. Nosotros la llamamos mundo y tenemos derecho a llamarla así, porque ella es lo que se encuentra en la epojé y, sin embargo, no se niega o pone en duda, sino que sólo entonces se saca de su anonimato originario a la luz" (J.Patočka, "Epojé y reducción", op.cit., p.248. Los subrayados son nuestros salvo en "epojé")

28 Cf. J.Patočka, "Forme-du-monde de l'expérience et expérience du monde", en Papiers Phénoménologiques, op.cit., pp211-25, especialmente la p.216-7-

${ }^{29}$ Dentro de los límites de este trabajo, hemos preferido no abordar el tema de la intersubjetividad. A modo de breve noticia, digamos que éste es uno de los temas concretos que aparecen igualmente en toda la obra de Patočka, ligados a la tematización de la subjetividad en su darse en el mundo. Al principio viene descrita, al modo husserliano, como intersubjetividad trascendental en la que aparece mundo. Posteriormente, es el mundo el que viene dado como presupuesto necesario para la intersubjetividad en tanto es un mundo común.

${ }^{30}$ Cf. "Přirozený svět jako filosofický problém", op.cit., pp.201-6. Un trabajo que aborda específicamente esta cuestión es "El espíritu y los dos estratos fundamentales de la intencionalidad", cf. "Der Geist und 
está, consiste en que en sus primeros años este horizonte venía subsumido en la subjetividad trascendental -es más, coincidía con la subjetividad trascendental ${ }^{31}-$, mientras que ahora el horizonte de horizontes señala el mundo como trascendental y condición de la subjetividad. En definitiva, el mundo -entendido como totalidad-, que desde muy pronto viene dado en el análisis, asume cada vez un protagonismo mayor hasta convertirse, en virtud de la epojé radical, en el trascendental y la condición para la donación del sujeto $^{32}$.

\section{5. ¿IRRUPCIÓN DE LA LIBERTAD O EMERGENCIA ANUNCIADA DE LA MISMA?}

\section{¿LA HISTORIA COMO RUPTURA O COMO EXPLICITACIÓN DE LO IMPLÍCITO?}

Una visión de conjunto de la obra fenomenológica de Patočka muestra, pues, una persistente meditación sobre el mundo y la existencia humana. Ciertamente, el aspecto que toma su meditación cambia notablemente de una época de su vida a otra. Es más, tampoco estas etapas son uniformes, sino que en ambas hay variaciones. Así, en sus primeros años le vemos oscilar entre una subjetividad trascendental entendida sobre todo como autoconciencia a una subjetividad marcada por la nota de la interioridad, donde la influencia de Bergson -ya presente, por lo demás, desde el principio $^{33}$ - se hace más presente; por otro lado, en sus últimos años, Patočka despliega su investigación en dos proyectos diferenciables, por más que luego quepa ver interrelaciones. Si la continuidad estriba en el tema fundamental de la subjetividad y el mundo, el cambio se explica, si nuestra interpretación es correcta, por el cambio en la noción de subjetividad, donde se pasa de tomarla como una realidad subsistente en sí misma y que alberga el infinito en sí, a considerarla - bajo el decisivo influjo de Heidegger- como el ámbito finito de

der zwei Grundschichten der Intentionnalität", en J.Patočka, Die Bewegung der menschlichen Existenz, Stuttgart, Klett-Cotta, 1991, pp.33-42.

${ }^{31}$ Cf.M. Bernard, "Le monde comme problème philosophique", art.cit., pp.358-9.

32 En realidad, este protagonismo del mundo estaba afirmado desde muy pronto, en su tesis de habilitación e incluso en escritos anteriores, por lo que la afirmación de su primacía respecto de la subjetividad era sólo cuestión de tiempo. Cf. R. Barbaras, "L'héroïsme de la philosophie devant le monde", en Jan Patočka, Liberté, existance et monde commun, op.cit., pp.87-106

${ }^{33}$ Encontramos ya un considerable resumen del pensamiento de Bergson y una valoración positiva de la misma en su tesis doctoral, cf. "Pojem evidence..", op.cit., 89-100 
aparición, de donación del mundo y del ser en el ente $e^{34}$.

Este tema, asimismo, es crucial para el conjunto de su filosofía, en todas las áreas trabajadas. Especialmente, juega un papel fundamental para uno de los temas más importantes de su reflexión sobre el hombre y la historia, a saber, la libertad y la responsabilidad. En concreto, la importancia para esta cuestión viene dada a partir de un rasgo concreto de la relación entre subjetividad y mundo, que es el de dos dinamismos complementarios: el contacto y la identificación con el mundo, por una parte; y la toma de distancia, por otra. Este doble dinamismo está presente en las descripciones de la donación del mundo, tanto las de sus primeros años como las posteriores ${ }^{35}$. Ambos dinamismos van en direcciones opuestas, ciertamente, pero su descripción indica también que se pertenecen mutuamente. El problema que se presenta entonces es el de si hay una continuidad entre el movimiento de contacto y distancia, o si más bien hay una dicotomía y oposición entre ambos. Esta dualidad de armonía y distanciamiento está presente igualmente en la teoría de los tres movimientos de la existencia humana, de manera que los dos primeros movimientos (arraigo y defensa) se corresponden con el contacto con el mundo y el tercero (penetración o verdad), con la toma de distancia ${ }^{36}$.

Asimismo, si la tensión entre anclaje y distanciamiento está presente en la teoría de los tres movimientos, igualmente lo está la pregunta sobre si habrá continuidad o cesura radical entre el contacto con el mundo y el distanciamiento. En el caso de la teoría de los tres movimientos, la pregunta se concreta en saber si el primer y el segundo movimientos se oponen

\footnotetext{
${ }^{34}$ Nos hemos centrado en su fenomenología. Sin embargo, el abandono del proyecto de fenomenología trascendental y la decisiva influencia de Heidegger son visibles en textos no fenomenológicos situados entre 1945 y 1960 . Estos textos tienen gran importancia porque permiten determinar la meditación que Patočka, sin duda, realizaba en fenomenología en estos años. Sin que podamos detenernos, señalemos brevemente, como elemento especialmente destacable, que, posteriormente a la primera crítica del subjetivismo de 1947 (con Eternidad e historicidad), Patočka muestra una profunda lectura de Heidegger en su proyecto de "platonismo negativo", según el cual lo verdaderamente central de la filosofía platónica era el tema del abismo (chorismós) y ello tiene su fundamento existencial en la negatividad que afecta a todo lo ente y que apunta a la diferencia ontológica. Cf. E.Tardivel, La liberté au príncipe..., op.cit., pp.53-80

${ }^{35}$ No entraremos aquí en el detalle de cómo esta descripción entre contacto y distanciamiento se da en los diferentes escritos de Patočka a lo largo de su vida. Nos permitimos remitir, para ello, a nuestro trabajo, ya citado, de la revista Pensamiento. Aquí podemos referir de sus primeros años la descripción de Lo interior y el mundo, cf. "Das Innere und die Welt", pp.42-6; y de sus años de madurez, cf. Body, Community, Language, World, pp.148-51.

${ }_{36}$ Para la presencia de la dualidad armonía/distanciamiento en la teoría de los tres movimientos de la existencia véanse las mismas páginas de Body, Community, Language, World citadas en la nota anterior.
} 
radicalmente al tercero o si hay continuidad entre ambos ${ }^{37}$. En Patočka hay elementos para las dos respuestas. Por una parte, se señala, en efecto, una divergencia radical entre el primer y segundo movimiento, por una parte, y el tercero por otra. En los dos primeros, estamos ligados al mundo y al mantenimiento de la vida, prevalece, pues, el motivo del contacto con el mundo ${ }^{38}$; en el movimiento de penetración o verdad, en cambio, tomamos distancia y nos situamos ante el mostrarse mismo del mundo y, con ello, ante el mundo como totalidad. Entre ambos movimientos parece haber una cesura radical, de manera que el tercer movimiento irrumpe en el tranquilo dominio de los dos primeros y se opone a los mismos; esta oposición, además, permanece, pues el hombre ha de luchar por mantenerse en esta situación de distanciamiento frente a la continua tendencia al declive ${ }^{39}$. Sin embargo, en otras descripciones el tercer movimiento parece, cuando menos, incoado en los dos primeros, como la presencia velada de la problematicidad y parece, asimismo, "preparado" por los dos primeros movimientos. Esta ambigüedad entre dicotomía o continuidad del anclaje y el distanciamiento, expresada en la teoría de los tres movimientos de la existencia humana, es especialmente visible en sus implicaciones para comprender la filosofía patočkiana de la historia. Así, en Ensayos heréticos se nos dice, por una parte, que el "mundo natural" de las sociedades prehistóricas es un mundo enteramente encerrado en el mantenimiento de la vida, en los dos primeros movimientos, frente a los que se contrapone posteriormente el tercer movimiento que, con el surgimiento simultáneo de la política y la filosofía, da comienzo a la historia. La historia, pues, marca una cesura radical respecto de la humanidad donde ella no se ha

\footnotetext{
${ }^{37}$ Cf P.Rodrigo, "Le problème de la cohérence de la théorie du mouvement chez Patočka : enracinement, percée et ébranlement du sens dans les Essais hérétiques" en Jan Patočka. Liberté, existence et monde commun, pp.161-77. Para una exposición en profundidad de la relación de los tres movimientos de la existencia, cf. E.Tardivel, La liberté au príncipe..., op.cit., pp.137-63; o bien R.Barbaras, "Le mouvement de l'existence chez Patočka", en Vie et intentionnalité, op.cit., pp.93-112.

${ }^{38}$ Hacia los años 70, Patočka apoyará este punto de vista en su lectura de Hannah Arendt. Ella está presente tanto en Ensayos heréticos como en los seminarios realizados a propósito de los mismos.

39 La caracterización del movimiento de trascendencia como una lucha que ha de ejercerse continuamente para no caer en el declive es otro tema recurrente en Patočka. El tercer movimiento, la libertad, la responsabilidad, el situarse ante el fenómeno, etc., no es una posesión pacífica, sino que lleva consigo la lucha por mantenerlo. Este tema está presente en fenomenología, filosofía de la historia, en estética, en la meditación sobre la historia checa, etc. Además, éste es uno de los sentidos -aunque no el único- en que ha de entenderse la afirmación del último de los Ensayos heréticos de que Heráclito tenía razón al decir que pólemos es el padre de todas las cosas. Cf. J. Patočka ,"Les guerres du XXe siècle et le XXe siècle en tant que guerre", en Essais hérétiques sur la philosophie de l'histoire, traducción de Erika Abrams, Paris, Verdier, 2007, 189-216 (pp.214-6)
} 
hecho aún presente ${ }^{40}$. Sin embargo, por otro lado, y en la misma obra, se dice igualmente que en la humanidad prehistórica está presente, de manera velada, el tercer movimiento por cuanto se barrunta la problematicidad. Los motivos de esta ambigüedad merecerían un análisis más detallado. En todo caso, y de modo preliminar, nosotros nos atrevemos a apuntar - siguiendo nuestra línea de estudio de los problemas que motivan, de fondo, un caminar filosófico- que la donación conjunta y co-pertenencia en tensión de contacto y distanciamiento, que localizamos en las descripciones patočkianas del primer estrato de la donación del mundo, están actuando en todo el despliegue del movimiento de la existencia.

En definitiva, puede decirse que el pensamiento de Patočka tiene como base una continua meditación sobre el mundo y la existencia humana. Considera constantemente cuál es su relación mutua a partir del hecho radical de la manifestación en el que ambos vienen dados; y en dicha meditación tiene lugar un cambio fundamental, por el que pasa de un marco trascendental subjetivo a otro donde el trascendental es el mundo entendido como esfera del aparecer, o "aparecer en cuanto tal", que se da a una subjetividad en cada caso finita; una subjetividad que, por otra línea de investigación, es movimiento de autorrealización inserta en el movimiento universal de autorrealización que es la ontogénesis del mundo. En cierto modo, el absoluto y lo infinito están presentes en todo momento, la diferencia estriba en que primero es la subjetividad la que lo alberga - diciendo incluso que encierra "la fuente de la vida eterna" ${ }^{41}$ - mientras que posteriormente la existencia se abre al infinito de la donación del mundo desde la apertura finita que es el movimiento de su existencia. Como indica acertadamente Filip Karfík, la subjetividad experimenta en Patočka una auténtica odisea, en la que absoluto se hace finito y donde la infinitud pasa de conquistarse yendo hacia el interior a alcanzarse entregándose al ser o al otro $^{42}$. A nosotros se nos plantea, como tarea de investigación,

\footnotetext{
${ }^{40}$ Cf. "Considérations préhistoriques" y "Le commencement de I'histoire", en Essais hérétiques..., op.cit., pp. 21-56 y 57-92. En estos dos textos, como han señalado algunos intérpretes, parece haber una vacilación en la misma descripción de Patočka, pues por un lado se dice que se trata de "El mundo sin problematicidad, , es un mundo donde el retirarse no es experimentado como tal [...] No conoce la experiencia del paso, la eclosión del ente como fenómeno..." (p.36) pero poco después que "hay un grado de esta vida en la evidencia que casi alcanza el umbral de la problematicidad" (p.38, subrayado mío). Tensiones similares pueden verse en otros lugares en estas páginas (por ejemplo, pp.64-6).

${ }^{41}$ Cf. "Das Innere und die Welt", op.cit., p.68.

42 Cf. F.Karfík, "Die Odysee des endlich gewordenen Absoluten. Patočkas systematische Versuche zwischen 1936 und 1964"; y "Unendlichwerden durch die Endlichkeit", en Unendlichwerden durch die Endlichkeit. Eine Lektüre der Philosophie Jan Patočkas, Würzburg, Königshausen\&Neumann, 2008,
} 
seguir los pasos el caminar filosófico de Patočka y, desde ahí, determinar si hemos de aceptar con él su filosofía de la existencia y del aparecer, o si más bien habremos de retomar los pasos de una fenomenología subjetiva -al modo de autores como Michel Henry-. En cualquier caso, sea cual sea nuestro acuerdo con sus tomas de postura, la obra de Patočka permanece como un eficaz revulsivo de cuestiones filosóficas fundamentales y un potente estímulo para reconsiderar momentos tan esenciales de la experiencia y tan fundamentales para la indagación filosófica como el hecho de que nuestra existencia se nos presente como dada en el mundo.

pp.32-54 y 71-81 respectivamente. Decimos "al ser o al otro" porque el mismo Patočka oscila entre uno y otro sentido de la trascendencia al infinito. En los años sesenta parece entender que la trascendencia tiene como expresión principal la entrega al otro; en cambio, posteriormente parece inclinarse por una interpretación más ontológica, donde la entrega se hace al misterio del ser en su diferencia del ente, de manera que se impide de este modo toda reducción del sentido de la existencia humana a un cálculo de entes. Con todo, no queda claro que Patočka renuncie explícitamente a la primera forma de trascendencia o que ambas sean rigurosamente incompatibles. Una presentación breve de la cuestión puede encontrarse en J.Frei, "Se dévouer à l'autre. Modalités de la trascendance chez Jan Patočka", en Jan Patočka. Liberté, existence et monde commun, pp.107-14. 